\title{
Agendas urbanas intersetoriais em quatro cidades de São Paulo'
}

\section{Intersetorial urban agendas based on four cities in São Paulo}

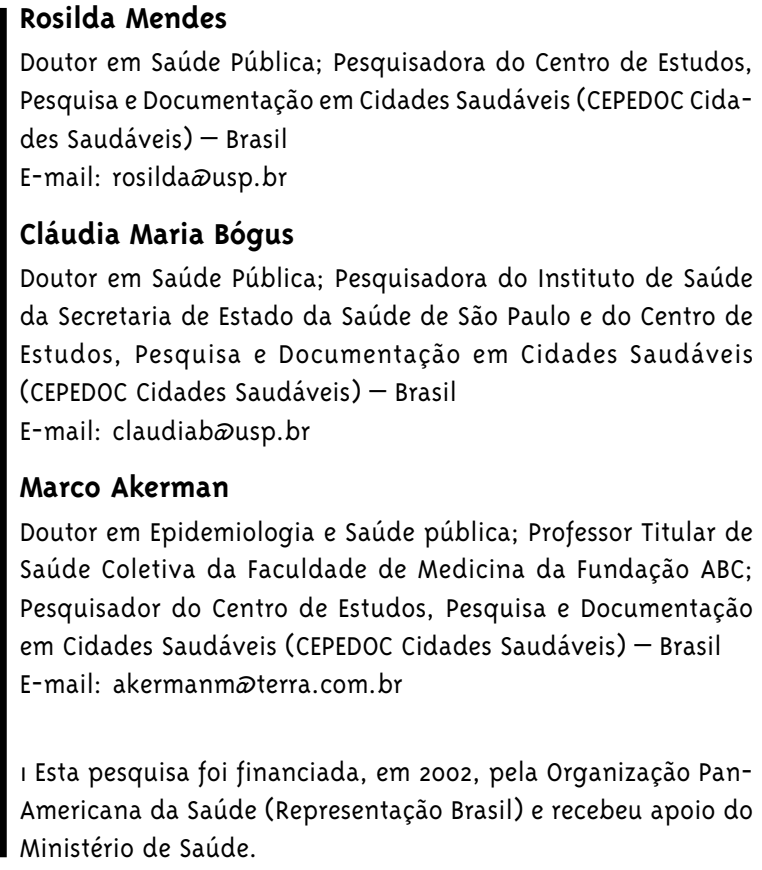

Doutor em Saúde Pública; Pesquisadora do Centro de Estudos, Pesquisa e Documentação em Cidades Saudáveis (CEPEDOC Cidades Saudáveis) - Brasil

E-mail: rosildaœusp.br

\section{Cláudia Maria Bógus}

Doutor em Saúde Pública; Pesquisadora do Instituto de Saúde da Secretaria de Estado da Saúde de São Paulo e do Centro de Estudos, Pesquisa e Documentação em Cidades Saudáveis (CEPEDOC Cidades Saudáveis) - Brasil

E-mail: claudiabðusp.br

\section{Marco Akerman}

Doutor em Epidemiologia e Saúde pública; Professor Titular de Saúde Coletiva da Faculdade de Medicina da Fundação ABC; Pesquisador do Centro de Estudos, Pesquisa e Documentação em Cidades Saudáveis (CEPEDOC Cidades Saudáveis) - Brasil

E-mail: akermanmळterra.com.br

I Esta pesquisa foi financiada, em 2002, pela Organização PanAmericana da Saúde (Representação Brasil) e recebeu apoio do Ministério de Saúde.

\section{Resumo}

Este artigo visa analisar o processo de implementação e avaliação de projetos de melhoria da qualidade de vida, do ponto de vista dos gestores municipais brasileiros, que têm buscado alternativas para a prática da gestão local. Optou-se por identificar a percepção de gestores, representantes do poder executivo local, prefeitos, secretários de governo e coordenadores, identificados como atores-chave importantes na implementação de projetos de melhoria da qualidade de vida em quatro municípios do Estado de São Paulo/Brasil. Foram feitas entrevistas individuais e em profundidade, com roteiro semi-estruturado. A análise dos quatro projetos por melhoria de qualidade de vida na cidade mostra que o processo de municipalização e descentralização da gestão, que vem sendo impulsionado no Brasil, vem contribuindo para definir uma política local mais autônoma, para criar novas instâncias de decisão, incorporando práticas de controle social e de democratização da gestão. Porém, ainda existem muitas limitações no que se refere à incorporação da avaliação, como elemento retro-alimentador, no planejamento das ações e da prática participativa.

Palavras-chave: Qualidade de vida, Gestão municipal, Intersetorialidade, Avaliação de projetos. 
Abstract

This article is designed to analyze the process of implementing and evaluating projects to improve quality of life, based on the view of Brazilian municipal managers who have sought local management alternatives. It was chosen to identify the perception of managers, representatives of local executive authorities, municipal secretaries and coordinators, all identified as key players in implementing quality of life improvement projects in four cities in the State of São Paulo/Brazil. Individual in-depth interviews were carried out using a semi-structured guide and comparative analysis. The analysis of the four projects aiming at improving quality of life in the city shows that the management decentralization processes, which are currently encouraged in Brazil, have contributed to define a more autonomous local policy and create new decision-making foro, incorporating social control practices and management democratization. However, there are many limitations regarding inclusion of assessment as a feedback to planning actions and participatory practice.

Key Words: Quality Of Life, Local Management, Intersectorial Action, Project Evaluation.

\section{Introdução}

Nos dias de hoje, podemos observar a existência de diversos movimentos nas diferentes regiões do país e do mundo, que têm por objetivo a melhoria da qualidade de vida e o aumento do potencial de articulação entre governos e grupos locais no desenvolvimento e fortalecimento de políticas de relevância social (Werna, 1996; Westphal, 2000; Mendes 2001). A complexidade dos problemas urbanos contemporâneos e as possibilidades oferecidas pelas cidades para a realização de projetos de melhoria da qualidade de vida fazem com que muitas das cidades brasileiras insiramse em movimentos por melhores condições de vida, filiando-se a distintas agendas sociais, como Cidades/ Municípios Saudáveis e Agenda 21, entre outros.

Essas propostas, guardadas suas especificidades, pressupõem pessoas vivendo em um dado território, enfrentando problemas concretos que demandam novas formas de gestão local, baseadas na ação intersetorial, na participação social e na eqüidade para a consecução dos objetivos da qualidade de vida.

Há uma tendência global de descentralização das políticas sociais e, conseqüentemente, de descentralização e municipalização de recursos e programas. No Brasil, o processo de descentralização, iniciado nos anos 1980, tem uma estreita relação com os processos de democratização política (Carvalho, 1990). Essa descentralização política oferece perspectivas interessantes. Uma das vantagens é o fato de que as políticas locais podem integrar os diferentes setores e articular os diversos atores (Dowbor, 1999). Outro aspecto refere-se ao papel desempenhado pelas autoridades locais, ou gestores sociais, que passam a ter uma importante função na definição de políticas e na execução de programas que beneficiam a população da cidade (Campagnone, 1990 ).

Esses gestores podem ser considerados pontos focais para a coordenação das ações intersetoriais, pois no nível local é possível unir a coletividade em torno de problemas específicos e buscar soluções próprias, convertendo o esforço conjunto em ações voltadas para a melhoria da qualidade de vida. Ao gestor municipal cabe um papel central na efetivação dessa lógica de governo com reorientação do desenho institucional, potencializando a ação comunicativa entre dirigentes, profissionais e população. Para isso, é fun- 
damental desenvolver uma dinâmica de aprendizagem e inovação, cujo primeiro passo deve ser a capacidade crescente de adaptação às mudanças.

A implementação de políticas sociais com esse enfoque implica na superação de políticas fragmentadas e o privilégio dado a projetos articulados, com o compartilhamento de informações e permeáveis à participação do cidadão, tanto no planejamento quanto na avaliação de ações e serviços.

Podemos observar como tem sido dada pouca importância ao acompanhamento das ações desenvolvidas para verificar a sua efetividade em termos de impacto social. A avaliação é um processo formativo que contribui para o fortalecimento das capacidades dos indivíduos, das comunidades e das instituições, desde que favoreça a aglutinação crítica dos atores sociais envolvidos por meio de um processo participativo. A questão, então, é fazer do processo avaliativo um instrumento para detectar "o porquê de o projeto ser ou não efetivo”, em uma localidade específica, e não fazer dele um exercício meramente científico, que busca identificar experiências bem-sucedidas, factíveis de replicação ou descartar aquelas malsucedidas, a partir do que é ou não efetivo (Akerman e col., 2002).

Quanto mais freqüentes as avaliações e quanto mais elas representarem um processo e não um evento único isolado, maior a oportunidade de que todos os envolvidos nos projetos e/ou programas em questão aprendam a trabalhar com os resultados obtidos. Os gestores, formuladores de políticas e tomadores de decisão poderão decidir com mais informações relevantes; os trabalhadores poderão melhorar o seu próprio desempenho e o dos projetos; os financiadores poderão analisar se seus investimentos estão sendo apropriados e a população, se os programas são adequados às suas demandas (Malik e Schiesari, 1998).

Outro aspecto importante no sentido de valorizar os processos avaliativos é que eles, além de demonstrarem a efetividade de procedimentos que promovam saúde e qualidade de vida, devem contribuir para o aprendizado, a ação e a transformação de práticas sociais (Wallerstein, 1992).

Esse artigo procura analisar o processo de implementação e avaliação de projetos de intersetoriais, que visam à melhoria da qualidade de vida, sob a ótica de gestores municipais, que têm buscado alternativas para a prática da gestão local.

\section{Metodologia}

Optou-se por identificar a percepção de gestores que ocupam posições de decisão, representantes do poder executivo local, prefeitos, secretários de governo e coordenadores, identificados como atores-chave importantes na implementação de projetos de melhoria da qualidade de vida em quatro municípios do estado de São Paulo/Brasil (Tabela 1). Foram feitas entrevistas individuais e em profundidade, com roteiro semiestruturado. 0 critério para seleção desses informantes não foi numérico, já que, do ponto de vista da metodologia qualitativa, a amostra ideal é aquela capaz de refletir a totalidade nas suas múltiplas dimensões (Minayo, 1993; Ludke e André, 1986).

Foram selecionados dois municípios que adotam projetos de Cidades Saudáveis: Limeira e Bertioga, e dois outros que estão desenvolvendo projetos intersetoriais que se aproximam do ideário da Agenda 21: Santo André e Piracicaba (Tabela 2).

Os depoimentos colhidos nas entrevistas individuais tiveram seus conteúdos analisados tematicamente. Buscou-se abordar fundamentalmente os seguintes temas: atores protagonistas da adoção e implementação da iniciativa, e, percepção em relação ao processo avaliativo dos projetos.

\section{Tabela I - Caracterização das cidades quanto à população e à atividade econômica}

\begin{tabular}{|l|c|l|}
\hline Cidades & População & Principais Atividades Econômicas \\
\hline Bertioga & 309.031 & Turismo, Comércio e Construção civil \\
\hline Limeira & 248.632 & Indústria de bijouterias, Autopeças e Turismo de negócios \\
\hline Santo André & 644.135 & Indústria, Comércio e Serviços \\
\hline Piracicaba & 328.312 & Agricultura: cana-de-açúcar e Indústria \\
\hline
\end{tabular}

Fonte: IBGE, 2000 
Tabela 2 - Informantes entrevistados por cidade

\begin{tabular}{|c|c|c|}
\hline Cidades & Projetos & Entrevistados \\
\hline Bertioga & Bertioga Município Saudável & Secretário adjunto e Secretária da Educação \\
\hline Limeira & Limeira Saudável & $\begin{array}{l}\text { Prefeito atual, Ex-prefeito, Coordenadora atual do projeto, } \\
\text { Ex-coordenadora do projeto e Secretário da Saúde }\end{array}$ \\
\hline Santo André & Santo André Cidade Futuro & Secretária Municipal do projeto e Secretário da Saúde \\
\hline Piracicaba & Piracicaba 2010: realizando o futuro & Secretário Executivo do projeto e Secretário da Saúde \\
\hline
\end{tabular}

\section{Resultados e Discussão}

\section{Atores protagonistas da adoção e implementação da iniciativa}

No Brasil, os projetos Cidades Saudáveis são recentes e tiveram início nos anos de 1990, quando algumas universidades e instituições apoiaram e incentivaram a discussão e o desenvolvimento desses projetos, visando à formação de uma rede brasileira, como uma estratégia que possibilitasse à ampliação do movimento no país (Mendes, 200o).

No caso das iniciativas, analisadas nesta pesquisa, e que adotaram a estratégia de Cidades Saudáveis como referência, confirmou-se a tendência de que instituições acadêmicas e organismos internacionais e nacionais do setor da saúde induziram o seu surgimento e implementação. Essa "indução" caracterizouse pela influência de universidades e outros atores externos, tais como: a Organização Pan-Americana da Saúde e o Ministério da Saúde, nos projetos. Isso determinou o protagonismo e a liderança inicial do setor da saúde.

Westphal e Mendes (2000) reiteram que, construir no interior de um governo, a marca Cidade Saudável não é uma questão fácil, já que, muitas vezes, o adjetivo "saudável" parece relacionar-se exclusivamente à saúde, o que gera dificuldades com as outras secretarias de governo. Isso reafirma a necessidade de que esses projetos sejam articulados com outras forças políticas e instâncias de controle social.

A Faculdade de Saúde Pública da Universidade de São Paulo (FSP/USP) acompanha o Projeto Bertioga Município Saudável (B) desde o ano de 1999, oferecendo apoio técnico e realizando projetos de pesquisas. O Projeto Limeira Saudável (L) conta com o apoio técnico da Pontifícia Universidade Católica de Campinas (PUC Campinas).
A percepção dos atores dessas duas cidades ilustra este aspecto, nas seguintes falas:

(L) Essa iniciativa veio para a Secretaria da Saúde, em meados de 98, através da PUC Campinas, que dava uma assessoria em outras áreas e foi ela que nos trouxe essa idéia do Município Saudável. O então secretário ficou muito interessado, começamos a conversar sobre isso, sobre essa idéia, sobre esse estilo de governar (...) o prefeito se empolgou muito, se entusiasmou, achou isso muito interessante (...) e foi assim que começou.

(B) O trabalho junto com a Universidade é importante. $O$ diagnóstico realizado em conjunto foi bem feito, todos os levantamentos, os grupos e as oficinas realizadas nos bairros da cidade. Foram elencadas as necessidades e as prioridades (...) Ter uma entidade, como a USP, como parceira, é um ótimo marketing para a cidade. Do ponto de vista político temos que explorar esta parceria

Ao mesmo tempo em que esse tipo de assessoria pode ser benéfica para os projetos, é preciso questionar qual a sua implicação quanto à sustentabilidade deles, ou seja, é possível a continuidade dos projetos após o afastamento das instituições de apoio externas? Os atores locais sentem-se fortalecidos e suficientemente articulados para isso?

Nesses dois municípios, a aproximação com a proposta foi justificada pelo seu potencial de organizar ações, com intenção de melhorar a qualidade de vida, para além do setor da saúde que tem sido, em muitos casos, o impulsionador do processo. Também houve referência significativa ao fato de representar uma forma alternativa de gestão local, com forte ênfase na intersetorialidade e na participação social, que são os principais componentes da proposta de Cidades Saudáveis (Westphal e Mendes, 200o, Santos e Westphal, 1999). 
(L) O que atraiu foi a questão de ser uma maneira diferente, inovadora de se trabalhar a qualidade de vida, não só dentro da Secretaria da Saúde mas num todo, na Prefeitura como um todo, envolvendo todo mundo (...)

Ao mesmo tempo, é interessante ressaltar que hoje em dia o setor saúde não atua mais como protagonista nesses municípios e que a adoção da proposta não implicou em reorientação dos serviços de saúde, dentro de uma perspectiva mais integral de saúde, que também está prevista na proposta de Cidades Saudáveis .

Em Limeira, por exemplo, a Secretaria de Saúde foi a que começou os primeiros contatos com a PUC Campinas. No início, houve resistência no secretariado, pelo entendimento de que se tratava de um projeto específico daquele setor. Apenas quando o prefeito assumiu a iniciativa como um projeto de governo houve a articulação com as outras secretarias municipais.

Nas outras duas iniciativas, Santo André Cidade Futuro (SA) e Piracicaba 2010: realizando o futuro (P), o governo local foi o grande indutor do processo de adoção e implementação. 0 papel de liderança concentrou-se desde o início no prefeito, afastando-se da liderança de um setor específico do governo, com forte apoio da sociedade civil, ou teve como base fundamental a sociedade civil exclusivamente, incluindo o setor privado.

Nesses municípios, o setor da saúde participou das iniciativas não como indutor, mas como integrante do processo. Nesse caso, as propostas que poderiam ser executadas pelo setor da saúde foram definidas no processo mais amplo de planejamento estratégico para o município.

(SA) "Tem um longo antecedente. O prefeito de Santo André sempre teve uma visão estratégica da cidade, não desenhada, mas uma proposta de pensar estrategicamente a cidade.

(P) (...) para a formação da ONG, nós obtivemos recursos das empresas. Temos aí uma lista dos patrocinadores que até então ajudaram e nos deram recursos monetários para que nós pudéssemos executar o plano. Daqui para frente, o que nós estamos pedindo aos sócio-mantenedores é manter a ONG. A despesa da ONG é pequena e os projetos em si não serão patrocinados pela ONG. Cada projeto, cada grupo de impulsão que vai trabalhar num projeto seja ele do governo, da sociedade civil seja ele um mix, esse gru- po tem que olhar o projeto como um todo, onde eu vou buscar recursos para fazer isso.

Nesses casos, as iniciativas tiveram como ponto de partida a identificação da necessidade de construção de uma visão estratégica de cidade, tendo como referência, explícita ou não, a proposição da Agenda 21 na busca de um modelo alternativo para o desenvolvimento integrado e sustentável (econômico, social e ambiental), elegendo um problema aglutinador que permitisse a mobilização social e, assim, privilegiando a participação social (Sorrentino e Tassara, 1999; Junqueira 200o).

(SA) Ele cria um grupo com empresários, sindicalistas, produtores culturais, representantes da diocese, da igreja católica, enfim um mix de pessoas da comunidade e com esse grupo ele começa a discutir os problemas de Santo André, tinha a crise que já abatia a cidade, a crise econômica (...) e começa a discutir com essas pessoas possibilidades, alternativas possiveis para o futuro dessa cidade (...) em agosto de 1992 nós organizamos o $A B C$ Ano 20oo, um seminário cuja tônica era discutir o futuro do $A B C$ não era nem Santo André, naquele momento era do $A B C$ Ano 20oo, a ótica era a questão econômica.

(P) (...) a iniciativa surgiu da necessidade de você ter um planejamento mais a longo prazo (...) Porque vocêvai criar uma visão de longo prazo, coisa que nós sentimos muito a falta hoje na administração pública. Você não tem visão a longo prazo, você tem projetos acontecendo de acordo com as necessidades (...) é difícil entender onde você quer chegar, onde Piracicaba vai chegar.

Ainda com relação ao processo de implementação das iniciativas, identificou-se, em todos os casos analisados, forte mobilização social. Estudos têm apontado a importância do protagonismo da sociedade civil, por meio de grupos organizados, pois são eles que vivem e sentem os problemas da cidade no seu cotidiano. As soluções são mais reais e efetivas se discutidas em um fórum aberto e democrático (Westphal, 1997; Ruiz, 1998).

A principal diferença entre as iniciativas investigadas relaciona-se com o foco de tal mobilização. Nos municípios de Bertioga e Limeira, a mobilização social é dirigida para a proposição e realização de ações, de acordo com as demandas definidas como prioritárias; nos municípios de Santo André e Piracicaba, essa 
mobilização volta-se para a elaboração de um planejamento estratégico integrado.

A mobilização social para ações priorizadas é uma forma de ampliar as capacidades que cada setor tem ao atuar isoladamente e estimular a realização de projetos integrados, que se concretizem em propostas e ações específicas, tais como: "Bairro Saudável”, em Limeira, e "Projeto Guarani" e a "Praça é Nossa”, em Bertioga.

Estudo realizado em Bertioga com o objetivo de analisar o processo participativo na experiência de implantação do Projeto Bertioga Município Saudável constatou alto grau de participação social, impulsionada pela utilização de metodologias participativas. Isso permitiu o envolvimento de representantes de todos os segmentos sociais e iniciou um processo de mobilização mais autônomo, ainda que incipiente (Arai, 2002; Comaru, 2004).

A mobilização social para o planejamento estratégico integrado tem como objetivo planejar e construir uma cidade para o futuro e dirige-se para a elaboração de macroprojetos que permitam e contemplem ações nos vários setores, como as 450 ações, no caso do projeto Santo André Cidade Futuro, e 52 macroprojetos, no caso do Piracicaba 2010: realizando o futuro.

(SA) Estabelecemos sete eixos: desenvolvimento econômico, desenvolvimento urbano, qualidade ambiental, inclusão social, educação, reforma do Estado, incluindo aí financiamento, participação popular, modernização administrativa e relação com o legislativo, e identidade cultural. (...) Esses grupos de trabalho são até hoje a espinha dorsal do projeto. No primeiro momento eles trabalharam diagnóstico, diretrizes e métodos. Para cada um dos temas, o segundo momento, tratou de estabelecer com maior precisão quais eram as metas para cada tema e no terceiro momento, que finalizou agora, estabelecemos um conjunto de ações para cada meta.

De forma geral, há um consenso quanto à dificuldade de dar visibilidade às iniciativas. Em todos os casos a repercussão é maior no início da implementação.

Em Bertioga e Limeira, têm sido utilizados espaços na mídia local (jornal, rádio e televisão). Em função de características específicas de cada uma das iniciativas, em Limeira, a proposta é veiculada como uma ação de governo e, em Bertioga, como ação da sociedade civil. Em ambos os casos estão sendo organizadas, atual- mente, Comissões de Comunicação e Marketing.

(B) No princípio houve maior publicidade, através da mídia local: jornal, rádio comunitária, mas que tem pouca abrangência, ou mesmo através da TV educativa. Pode-se dizer que o Projeto Bertioga Município Saudável já apareceu mais na mídia, mas hoje diminuiu. Não se pode dizer que falte espaço na mídia. A mídia local tem pouco assunto para transmitir.

(L) (...) então nós temos alguma divulgação esporádica da mídia, nós temos um programa que semanalmente sai nos jornais aí que é a notícia da Prefeitura então nós colocamos, que é alguma realização no bairro, o que tem sido feito, então nós procuramos $e$ isso tem despertado a comunidade, que estão reivindicando, mas por iniciativa própria, pelo interesse da mídia de uma forma geral não está havendo muita divulgação (...).

A iniciativa de Santo André adquiriu visibilidade local, estadual, nacional e internacional por meio da realização de eventos com participação de vários consultores nacionais e internacionais, que trouxeram a experiência de outras localidades, e da sua inserção ao projeto Merco-Cidades (rede de cidades pertencentes ao Mercosul que des envolvem planejamento estratégico-participativo).

A experiência da iniciativa de Santo André mostrou que projetos e propostas que levem em conta a cidade como um todo possuem um alto potencial de visibilidade local, estadual, nacional e internacional.

(SA) (...) acabamos estruturando junto com várias pessoas das prefeituras da região um Seminário Internacional da Câmara (Regional do $A B C$ ) que era o primeiro movimento da câmara regional. Para esse seminário nós trouxemos experiências de reconversão econômica da Europa e dos Estados Unidos, então nós trouxemos uma experiência da Alemanha que era da Alemanha Oriental do Muro de Berlim e que eles estavam discutindo a econômica local uma experiência da recuperação da Galícia na Espanha, experiência no nordeste da Inglaterra e uma experiência sobre Detroit. $E$ aínós colocamos junto com esses palestrantes debatedores brasileiros, pessoas respeitadas nessa área de atuação.

A iniciativa de Piracicaba obteve visibilidade e repercussão por meio do site do projeto e da distribuição do documento contendo os 52 macroprojetos desenhados. 


\section{Percepções em relação aos processos avaliativos dos projetos}

O limitado sucesso de muitos programas e o desconhecimento dos efeitos e impactos produzidos pelos investimentos realizados nas políticas públicas e nos programas e projetos de desenvolvimento social têm feito com que os governos, as organizações não- governamentais e as agências nacionais e internacionais reivindiquem cada vez mais a inclusão de metodologias de monitoramento e avaliação de processo, resultados e impacto dos programas implementados (Buvinich, 1999; Akerman e col.; WHO, 1998).

Nesse estudo, as entrevistas mostraram que a questão da avaliação é uma preocupação presente, mas que a sistematização desse tipo de atividade é muito incipiente. Os estudos realizados por Buvinich (1999) demonstram que essa é uma característica comum aos projetos e programas desenvolvidos na América Latina, onde são raros aqueles que aprofundam a avaliação, para demonstrar efeitos e impactos. A maior parte deles restringe-se apenas à descrição das discrepâncias entre o realizado e o programado, e entre os recursos implementados e as coberturas alcançadas.

Nos Projetos Bertioga Município Saudável e Limeira Saudável a avaliação tem apoio externo de equipes técnicas de universidades, embora em Bertioga exista avaliação do projeto na Comissão Intersetorial através de um Guia de Avaliação Participativa de Municípios Saudáveis (Wallerstein e col., 1997), que vem sendo elaborado pela OPAS para ser aplicado na região das Américas. Não há o reconhecimento, por parte da equipe de governo, de que esta atividade de avaliação tenha sido incorporada na tomada de decisões, por parte das autoridades locais.

(B) a avaliação das políticas públicas da cidade é realizada através do prefeito, um conhecedor profundo da comunidade. Isto indica a resposta ao trabalho da administração. Ele tem claro um diagnóstico. Uma única vez que se consultou uma pessoa de fora da cidade, que utilizou um instrumento sistematizado de pesquisa, concluiu-se que a sua avaliação era igual à percepção do prefeito (...) o prefeito é da área de saúde e sabe dos problemas da área.

No caso de Limeira, as avaliações que aconteceram até o momento são caracterizadas como pontuais e muitas delas envolveram apenas a equipe técnica com a participação de assessoria externa. A avaliação com o grupo local onde o projeto "Bairro Saudável" tem sido desenvolvido utilizou instrumentos da área de qualidade total. Um dado importante é o início da estruturação de um Observatório da Qualidade de Vida, que pretende definir indicadores comparativos com a região para suprir a falta de dados disponíveis, o que dificulta análises quanto aos resultados e impacto das ações desenvolvidas.

(L) Agora, essa questão dos dados numéricos, na verdade o que nós estamos fazendo é montando, nós estamos numa fase de comparar Limeira com municípios com as mesmas características, então a gente vai comparar, ver, ter uma visão mais panorâmica, (...) conseguir olhar a questão da qualidade de vida em Limeira por bairros ou regiões, ou seja, então o que nós temos hoje é a situação vamos dizer atual, e como o Prefeito colocou daqui alguns meses a gente vai poder estar trabalhando no sentido de ter essa comparação. É um trabalho mais técnico, mais interno (...).

Nos projetos Santo André Cidade Futuro e Piracicaba 2010: realizando o futuro é possível caracterizar a avaliação como interna. Em Santo André, existem indicadores para o desenvolvimento de avaliações do projeto como um todo. Esses indicadores foram desenhados durante o processo de planejamento participativo, que tem construído a própria iniciativa. No caso de Piracicaba, o projeto encontrava-se na fase de iniciar a implantação das ações que compunham os 52 macroprojetos elaborados. Com o objetivo de gerenciar essas ações e projetos foram organizados grupos de impulsão que seriam responsáveis pelo monitoramento, através de um software específico.

O projeto Santo André Cidade Futuro não tem a pretensão de ser a iniciativa que transformará a cidade, mas pretende ser uma estratégia de articulação e integração de princípios, diretrizes e instrumentos, previamente concebidos, no bojo do programa de governo. Para cada uma das ações há metas programadas, criando-se, portanto, bases para exercícios avaliativos.

(SA) Olha é menos uma coisa de número mas a fala das pessoas, dá para sentir que as pessoas que participam do projeto se sentem recompensados por participar".

É importante assinalar que, em Santo André, outro projeto prioritário do governo municipal, o "orçamento participativo", incorpora na sua programação os 
mesmos nove eixos temáticos do projeto Cidade Futuro. Pode-se supor que existem elementos para se desenvolver, posteriormente, uma avaliação mais sistemática do projeto.

\section{Considerações Finais}

A análise dos quatro projetos que visam à melhoria da qualidade de vida nas cidades mostra o grande desafio da construção de uma cidade saudável ou de uma cidade do futuro, já que isso implica em mudar as tradicionais formas de gestão que estão histórica e culturalmente consolidadas. A criação de uma nova cultura política exige que se definam novas formas de relação entre os diferentes níveis de governo e de novas formas de cooperação e articulação no desenvolvimento de uma nova mentalidade, de novas habilidades e de estratégias para uma atuação conjunta e compartilhada.

Como vimos, os problemas das cidades são de grande complexidade e o seu enfrentamento implica no estabelecimento de parcerias e na definição de ações intersetoriais e integradoras, que pressupõem a articulação de planos e o compartilhamento de informações para o desenvolvimento de projetos amplos, que permitam e agreguem a participação dos diferentes setores envolvidos na solução dos problemas urbanos.

A qualidade de vida, como produto social, resulta de uma ação pactuada pelo governo. Os gestores são atores políticos condutores do desenvolvimento de políticas públicas de relevância social na cidade. Mas não são os únicos. Todas as políticas públicas que tenham, como critério decisório, a capacidade de modificar a qualidade de vida da população devem necessariamente ser gestadas a partir de um planejamento conjunto e estratégico que represente a união de todos os setores. Nesse sentido, é fundamental que se incorpore no planejamento a idéia da avaliação como elemento retro-alimentador de todo o processo, permitindo as necessárias correções de estratégias.

A realização de planejamentos conjuntos e estratégicos é uma tarefa difícil de ser viabilizada, como mostram os depoimentos dos gestores municipais entrevistados, mas pode ser viabilizada dentro de uma perspectiva processual e gradativa.

\section{Agradecimentos}

Agradecemos a colaboração dos gestores e profissionais das prefeituras de Bertioga, Limeira, Santo André e Piracicaba, pelas informações prestadas, sem as quais esse trabalho não poderia ter sido realizado.

\section{Referências}

AKERMAN, M. et al. Avaliação em promoção da saúde: foco no município saudável. Revista de Saúde Pública, São Paulo, v. 36, n. 5, p. 638-646, 2002.

ARAI, V.J. Análise de um processo participativo na experiência de implantação de um projeto município saudável. São Paulo, 2002. Dissertação (Mestrado) - Faculdade de Saúde Pública, Universidade de São Paulo.

BUVINICH, M.R. A importância da avaliação de programas e projetos sociais. Vivavoz / Fundação Banco do Brasil, n. 8, set. 1999.

CAMPAGNONE, M.C. Gerente municipal: um profissional da gestão local. In: CENTRO DE ESTUDOS E PESQUISAS DE ADMINISTRAÇÃO MUNICIPAL (CEPAM). O município no século XXI: cenários e perspectivas. São Paulo, 1999. p.25-38.

CARVALHO, G. Saúde: avanços e entraves ao processo de descentralização. In: CENTRO DE ESTUDOS E PESQUISAS DE ADMINISTRAÇÃO MUNICIPAL (CEPAM). O município no século XXI: cenários e perspectivas. São Paulo, 1999. p.135 - 148.

COMARU, F.A. Políticas de habitação e desenvolvimento urbano em municípios saudáveis: o caso de Bertioga - São Paulo. São Paulo, 2004. Tese (Doutorado) - Faculdade de Saúde Pública, Universidade de São Paulo.

DOWBOR, L. Tendências da gestão social. Saúde e Sociedade, São Paulo, v. 8, n. 1, p. 3-14, 1999.

JUNQUEIRA, R. G. P. Agendas sociais: desafio da intersetorialidade na construção do desenvolvimento local sustentável. Revista de Administração Pública, Rio de Janeiro, v. 34, n. 6, p. 117 -130, 2000.

LUDKE, M.; ANDRÉ, M.E.D.A. Pesquisa em educação: abordagens qualitativas. São Paulo: Editora Pedagógica Universitária, 1996. 
MALIK, A. M.; SCHIESARI, L. Qualidade na gestão local de serviços e ações de saúde. São Paulo: Faculdade de Saúde Pública da Universidade de São Paulo, 1998. (Série Saúde \& Cidadania, v. 3)

MENDES, R. Cidades saudáveis no Brasil e os processos participativos: os casos de Jundiaí e Maceió. São Paulo, 200o, Tese (Doutorado) - Faculdade de Saúde Pública, Universidade de São Paulo.

MENDES, R. Qualidade de vida nas cidades: o movimento por cidades saudáveis. Qualidade de Vida / Centro de Estudos Avançados em Economia Aplicada USP, São Paulo, v. 3, n. 26, p. 1 -4, 2001.

MINAYO, M. C. S. O desafio do conhecimento: pesquisa qualitativa em saúde. São Paulo: HUCITEC/ ABRASCO, 1993.

RUIZ, G. Análisis de la participación social en el movimiento municipios saludables en América Latina. Laval, 1998, Disertación (Maestría) - Faculté de Médicine, Université de Laval.

SANTOS, J. L.; WESTPHAL, M.F. Práticas emergentes de um novo paradigma de saúde: o papel da universidade. Estudos Avançados, São Paulo, v. 35, p. 71 88, 1999.

SORRENTINO, M.; TASSARA, E.T.O.. Educando para o desenvolvimento sustentável. In: CENTRO DE ESTUDOS E PESQUISAS DE ADMINISTRAÇÃO MUNICIPAL (CEPAM). O município no século XXI: cenários e perspectivas. São Paulo, 1999. p.185 - 190.
WALLERSTEIN, N.; MALTRUD, K.; POLACSEK, M. Participatory evaluation workbook for community initiatives. New Mexico: New Mexico Department of Health, Public Health Division, Healthier Communities Unit; 1997.

WALLERSTEIN, N. Powerlessness, empowerment, and health: implications for health promotion programs. American Journal of Health Promotion, Rochester Hills, v. 6, n. 3, p. 197 - 205, 1992.

WERNA, E. As políticas urbanas das agências multilaterais de cooperação internacional para países em desenvolvimento. Espaço e Debates, São Paulo, v. 39, p. 10-12, 1996.

WESTPHAL, M.F. Municípios saudáveis: aspectos conceituais. Saúde e Sociedade, São Paulo, v. 6, n. 2, p. 9 - 18, 1997.

WESTPHAL, M.F. O movimento de municípios saudáveis e a conquista da qualidade de vida. Ciência e Saúde Coletiva, Rio de Janeiro, v. 5, n. p. 39-51, 2000.

WESTPHAL, M. F.; MENDES, R. Cidade saudável: uma experiência de interdisciplinartidade e intersetorialidade. Revista de Administração Pública, Rio de Janeiro, v. 34, n. 6, p. 47 - 61, 2000.

WHO REGIONAL OFFICE FOR EUROPE. Health promotion evaluation: recommendations to policymakers. Copenhagen, 1998. 